\title{
Light-Induced Phase-Shifting of the Peripheral Circadian Oscillator in the Hearts of Food-Deprived Mice
}

\author{
Katsuhiko SAKAMOTO1)*, Koji KADOTA2), and Katsutaka OISHI3)* \\ ${ }^{1)}$ Neuroscience Institute, Morehouse School of Medicine, 720 Westview Dr. S.W., Atlanta, GA 30310-1495, \\ USA, ${ }^{2}$ Computational Biology Research Center, National Institute of Advanced Industrial Science and \\ Technology, Aomi Frontier Bldg. 17F, 2-43 Aomi, Koto-Ku, Tokyo 135-0064, and ${ }^{3}$ Clock Cell \\ Biology Research Group, National Institute of Advanced Industrial Science and Technology, \\ Central 6, 1-1-1 Higashi, Tsukuba 305-8566, Japan
}

\begin{abstract}
In the present study, we investigated the effect of fasting on photoentrainment of the peripheral circadian oscillator in the mammalian heart. Northern blotting showed that a single light pulse applied at an appropriate time in constant darkness, caused obvious phase-shifting in the circadian expression rhythm of the mammalian clock gene Period2 (mPer2) even in the hearts of food-deprived mice. Fasting did not significantly affect either the phase or the light-induced phase-shifts of the mPer2 rhythm. Although several studies of temporal feeding restriction have indicated that feeding is the dominant timing cue for mammalian peripheral oscillators, our findings suggest that feeding is not essential for mammals to induce phase resetting of the circadian oscillator in the heart.
\end{abstract}

Key words: circadian rhythm, clock gene, fasting

In mammals, the central clock that regulates most physiological and behavioral rhythms is located in the suprachiasmatic nucleus $(\mathrm{SCN})$ of the hypothalamus in the brain [7]. Many studies at the molecular level have revealed that the circadian oscillator in the $\mathrm{SCN}$ is driven by negative feedback loops consisting of the periodic expression of clock genes [7,8]. Several experiments on clock genes indicate the existence of an oscillatory system in peripheral tissues (such as the liver, kidneys, heart, etc.) that contains feedback loops similar to those in the SCN [7, 12]. Mammalian peripheral tissues maintain circadian expression of clock genes even in vitro $[1,16,17]$. However, peripheral oscilla- tors require the SCN to synchronize to daily light-dark cycles $[3,7,9,12,17]$. The master pacemaker SCN probably functions as a phase coordinator for peripheral clocks [12, 17].

Interestingly, recent studies of the mammalian circadian system have demonstrated that temporal feeding restriction can entrain circadian oscillators in peripheral tissues (including the heart) independently of the SCN master clock and environmental light cycle [2-4, 13]. Feeding time seems to be the dominant time cue for peripheral circadian oscillators. In mammals, temporal feeding patterns are imposed through $\mathrm{SCN}$-driven rest-activity cycles. Thus, peripheral oscillators may

*These authors contributed equally to this work.

(Received 23 March 2004 / Accepted 2 June, 2004)

Address corresponding: K. Sakamoto, Neuroscience Institute, MRC RM217, Morehouse School of Medicine, 720 Westview Dr. S.W., Atlanta, GA 30310-1495, USA 
be coupled to the SCN pacemaker primarily through feeding behavior $[8,12]$. However, little is known about the molecular mechanism by which feeding induces phase resetting of peripheral circadian oscillators.

Many researchers have extensively studied the molecular mechanism of the circadian oscillator in the mammalian heart $[4-6,10,11,14,18,19]$. In the mouse heart, about $10 \%$ of the mRNA population are expressed in a circadian manner [14]. Circadian expressions of these genes appear to be governed by the peripheral clock in the heart [12]. It is likely that circadian gene expression in peripheral tissues serves to optimize cellular physiology by sequestering chemically incompatible reactions to different time windows [12].

We showed that an appropriate light stimulus produces overt phase-shifts in the circadian mRNA expression rhythms of clock genes in the mouse heart [10]. In the present study, we investigated the effect of fasting on photoentrainment of the mammalian peripheral oscillator. We used Northern blotting to analyze light-induced phase-shifts in the circadian expression rhythm of the mammalian clock gene Period2 ( $m P e r 2$ ) in the hearts of food-deprived mice. Rhythmic expression of the clock gene mPer 2 is considered to play a critical role in the core mechanism of mammalian circadian oscillators [7].

Six-week-old adult male Balb/c mice were obtained from CLEA JAPAN, INC. (Tokyo) and were housed under a daily 12-h light/dark cycle (LD12:12, lights on 0:00-12:00 h) for two weeks as previously described [10]. The animals were then transferred to constant darkness (DD), and exposed to a 12-h light pulse ( 200 lux) at the time corresponding to subjective night (12:00-24:00 h) on the first day under DD (Fig. 1A), as previously described [10]. We identified phase-shifts of $m P e r 2$ mRNA on the second day after the light pulse, since we previously confirmed that $m P e r 2$ mRNA in the mouse heart does not undergo a light-induced phaseshift on the first day after exposure to light [10]. Fasting mice were deprived of food from the start of exposure to the light pulse, and maintained under fasting conditions. Control animals were allowed free access to food throughout the experiment. Autoclaved solid chow constituted the mouse diet in this study. Water was supplied ad libitum. Three individuals were sacrificed at each time point on the third day under DD, or two days after exposure to light. Tissues (i.e. the whole heart) were dissected, quickly frozen, and stored at $80^{\circ} \mathrm{C}$. All experiments proceeded in accordance with the European Communities Council Directive of 24 November 1986 (86/609/EEC).

We carried out Northern blotting to determine the temporal expression patterns of $m P e r 2 \mathrm{mRNA}$ in the heart. Total RNA ( $20 \mu \mathrm{g}$ per lane) isolated from each tissue was separated on gels, blotted onto a nylon membrane by passive capillary transfer and probed with a ${ }^{32} \mathrm{P}$-labeled random primed probe, as described [10]. The probe generated from a cDNA fragment of $\mathrm{mPer} 2$ (bases 1123-1830; GenBank accession number AF036893) was hybridized and detected as described [10]. Samples were normalized by comparison with the amount of glyceraldehyde-3-phosphate dehydrogenase mRNA (GAPDH; GenBank accession number M17701; data not shown).

A nonlinear least-squares (NLLS) MarquardtLevenberg algorithm was performed to fit a curve to the observations. We defined the function as $f(x)=M$ $+A \cos (2 \pi / T(x-\phi))$ and set four variables (M, MESOR (mean statistics of rhythm); A, Amplitude; T, Period and $\phi$, Acrophase) as the fit parameters. We verified the significance $(p<0.05)$ of the light-induced phaseshift by the absence of an overlap between two intervals $[\phi-1.96 S E, \phi+1.96 S E]$ with and without light exposure, where SE is the standard error of acrophase. Acrophase comparisons were performed by Welch's $t$ test and $p<0.05$ was considered statistically significant.

As described above (Fig. 1A), we applied a 12-h light pulse at subjective night on the first day under DD and produced phase-shifts in circadian rhythms of mice. The light pulse evoked an obvious phase-shift (a delay of $\sim 3.2 \mathrm{~h}$ ) of the mPer 2 mRNA rhythm in the heart of control animals with access to food ad libitum (Fig. 1B and C; Table 1), as reported [10]. Probably, the light stimulus reset the SCN master clock and then the SCN altered the phase of the peripheral oscillator $[8,12]$. Since peripheral oscillators are considered to be coupled to the SCN pacemaker basically through feeding behavior $[8,12]$, the heart oscillator in the control mice under ad libitum feeding might shift its phase primarily through the rhythmic feeding controlled by the light-induced phase-shifted SCN clock.

Interestingly, the $m P e r 2$ rhythm in food-deprived mice was as significantly phase-shifted by light as that of the control mice (Fig. 1B and C; Table 1). The 
(A)

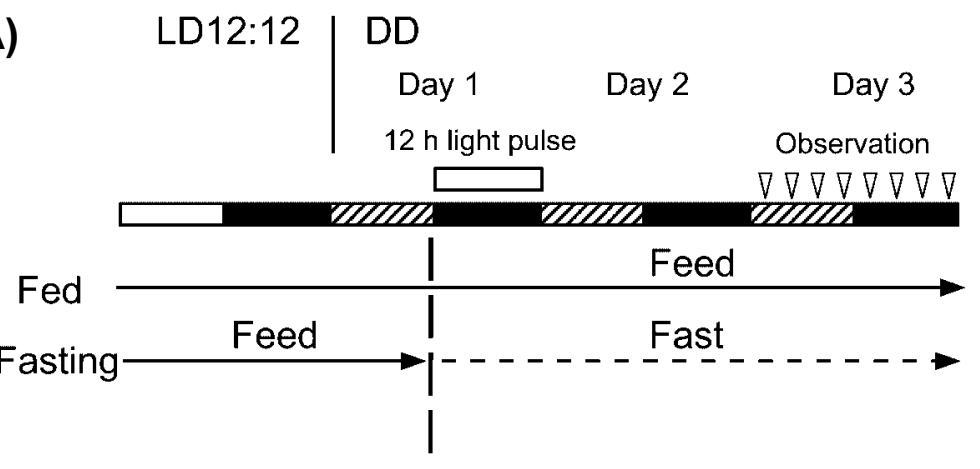

(B)

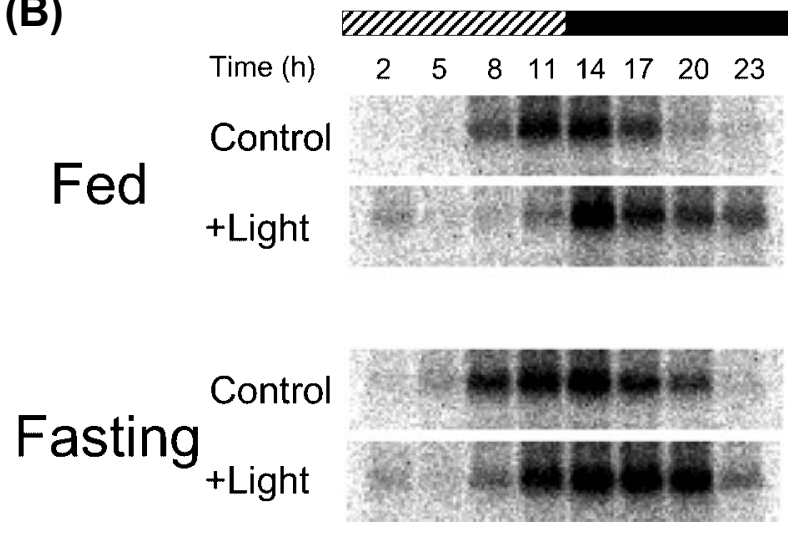

Fig. 1. Light-induced phase-shifts in circadian expression rhythm of $m P e r 2$ mRNA in hearts of food-deprived mice. (A) Schedule of phase-shift experiments. Mice were maintained under a daily 12-h light/dark cycle (LD12:12, lights on 0:00-12:00 h) for 2 weeks and then transferred to constant darkness (DD). A 12-h light pulse was applied to mice at the time corresponding to the subjective night on the first day of DD. Fasting mice were deprived of food from the beginning of the light pulse, and maintained under fasting conditions. Open bars, lights on; solid bars, lights off or subjective night under DD; striped bars, subjective day under DD; horizontal solid lines, feeding period; horizontal dotted line, fasting period; open triangles, sampling points. (B) Representative Northern blots of $m P e r 2$ mRNA in mouse heart. Each lane contained $20 \mu \mathrm{g}$ total RNA obtained from individual mice. Shaded bar, subjective lights-on; solid bar, subjective lights-off, on day 3 under DD. (C) Quantification of mPer $2 \mathrm{mRNA}$ levels from blots (B). Maximal value of mRNA level under ad libitum feeding (Fed) without light stimulus is expressed as $100 \%$. Open circles, group exposed to light; solid circles, control (no light pulse). Values are means $\pm \operatorname{SEM}(n=3)$. Non-linear least-squares (NLLS) MarquardtLevenberg algorithm determined a curve fit to the observations.
(C)

Fed

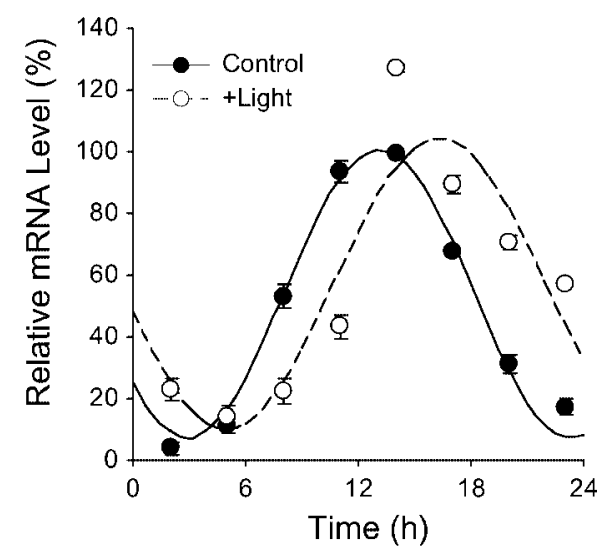

Fasting

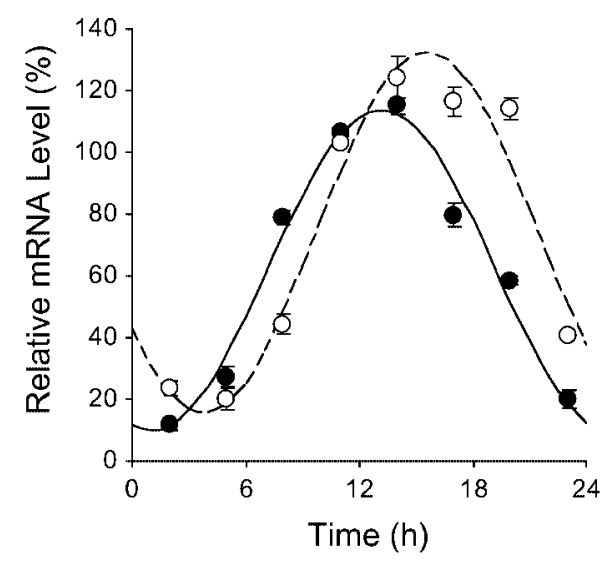

values from fasting and fed animals did not significantly differ. Furthermore, food deprivation did not significantly affect the acrophases of the $\mathrm{mPer} 2$ rhythm in the heart, regardless of exposure to the light pulse (Table 1). These findings suggest that fasting does not affect either the phase or light-induced phase-shifts of the circadian oscillator in the mouse heart. Therefore the present study implies that feeding is not essential for mammals to induce phase resetting of the peripheral oscillator in the heart. Although recent studies of temporal feeding restriction indicate that the most dominant entrainment pathway for peripheral clocks involves 
Table 1. Comparison of acrophases of the mPer2 rhythm in the heart between light-exposed and control groups in fed and fasting mice

\begin{tabular}{lccc}
\hline & Fed & Fasting & Fed vs. Fasting \\
\hline Control & $13.12 \pm 0.14$ & $13.17 \pm 0.16$ & $p>0.1$ \\
+Light & $16.32 \pm 0.41$ & $15.57 \pm 0.22$ & $p>0.1$ \\
Control vs. + Light & $p<0.01$ & $p<0.01$ & \\
\hline
\end{tabular}

Acrophases as peak times (hours) of $m P e r 2$ rhythm on the day 3 under DD determined using fitting curves described in Fig. 1C (for details see the Materials and Methods). Values represent means \pm SEM $(n=3)$. Acrophase comparisons performed by Welch's $t$-test and $p<0.05$ was considered statistically significant.

feeding behavior [12], the SCN might be able to synchronize the phase of peripheral oscillators even through signaling pathways that do not involve feeding. In mammals, the master clock SCN synchronizes peripheral circadian oscillators through direct and indirect signaling pathways such as humoral factors, neural connections and body temperature $[12,15]$. To identify the feeding-independent signaling pathways, further studies are required.

The present study on the heart showed that even in fasting animals a light stimulus causes significant phaseshifts of the peripheral circadian oscillator. However, this phenomenon may be found only in some tissues. Food-induced phase resetting proceeds faster in the liver than in the kidneys, heart, or pancreas [2]. The SCN appears to synchronize the phase of peripheral oscillators in different ways, and thus peripheral oscillators in some tissues may be entrained more tightly through feeding behavior. Actually the amplitude of light-induced phase-shifts in the hearts of fasting mice was somewhat smaller (not significantly, but $25 \%$ less) than that of control mice fed ad libitum (Fig. 1B and C; Table 1). This tendency could be explained if peripheral tissues receive fewer time signals because feeding-dependent signaling pathways are negated under food deprivation. Further elucidation of the resetting mechanism in peripheral oscillators should provide the basis for a comprehensive understanding of the mammalian circadian system.

\section{Acknowledgments}

We are grateful to Dr. Gianluca Tosini for his critical comments regarding the manuscript. This project was supported by a Grant-in Aid for Young Scientists (B) (15700492) to K. Oishi from the Ministry of Education, Culture, Sports, Science and Technology of Japan (MEXT).

\section{References}

1. Balsalobre, A., Damiola, F., and Schibler, U. 1998. Cell 93: 929-937.

2. Damiola, F., Le Minh, N., Preitner, N., Kornmann, B., Fleury-Olela, F., and Schibler, U. 2000. Genes Dev. 14: 2950-2961.

3. Hara, R., Wan, K., Wakamatsu, H., Aida, R., Moriya, T., Akiyama, M., and Shibata, S. 2001. Genes Cells 6: 269-278.

4. Minami, Y., Horikawa, K., Akiyama, M., and Shibata, S. 2002. FEBS Lett. 526: 115-118.

5. Oishi, K., Miyazaki, K., and Ishida, N. 2002. Biochem. Biophys. Res. Commun. 298: 198-202.

6. Portman, M.A. 2001. Circ. Res. 89: 1084-1086.

7. Reppert, S.M. and Weaver, D.R. 2001. Annu. Rev. Physiol. 63: 647-676.

8. Ripperger, J.A. and Schibler, U. 2001. Curr. Opin. Cell Biol. 13: 357-362.

9. Sakamoto, K., Nagase, T., Fukui, H., Horikawa, K., Okada, T., Tanaka, H., Sato, K., Miyake, Y., Ohara, O., Kako, K., and Ishida, N. 1998. J. Biol. Chem. 273: 27039-27042.

10. Sakamoto, K. and Ishida, N. 2000. Eur. J. Neurosci. 12: 4003-4006.

11. Sakamoto, K., Oishi, K., Nagase, T., Miyazaki, K., and Ishida, N. 2002. Neuroreport 13: 1239-1242.

12. Schibler, U., Ripperger, J., and Brown, S.A. 2003. J. Biol. Rhythms 18: 250-260.

13. Stokkan, K.-A., Yamazaki, S., Tei, H., Sakaki, Y., and Menaker, M. 2001. Science 291: 490-493.

14. Storch, K.F., Lipan, O., Leykin, I., Viswanathan, N., Davis, F.C., Wong, W.H., and Weitz, C.J. 2002. Nature 417: 78-83.

15. Terazono, H., Mutho, T., Yamaguchi, S., Kobayashi, M., Akiyama, M., Udo, R., Ohdo, S., Okamura, H., and Shibata, S. 2003. Proc. Natl. Acad. Sci. USA 100: 6795-6800.

16. Yamazaki, S., Numano, R., Abe, M., Hida, A., Takahashi, R.-I., Ueda, M., Block, G. D., Sakaki, Y., Menaker, M., and Tei, H. 2000. Science 288: 682-685.

17. Yoo, S.-H., Yamazaki, S., Lowrey, P.L., Shimomura, K., Ko, C.H., Buhr, E.D., Siepka, S.M., Hong, H.-K., Oh, W.J., Yoo, O.J., Menaker, M., and Takahashi, J.S. 2004. Proc. Natl. Acad. Sci. USA 101: 5339-5346.

18. Young, M.E., Razeghi, P., and Taegtmeyer, H. 2001. Circ. Res. 88: 1142-1150.

19. Young, M.E., Wilson, C.R., Razeghi, P., Guthrie, P.H., and Taegtmeyer, H.J. 2002. Mol. Cell. Cardiol. 34: 223-231. 